\title{
Dual recycling for GEO 600
}

\author{
A. Freise for the GEO 600 team \\ Albert-Einstein-Institut Hannover, \\ Callinstr. 38, 30167 Hannover, Germany
}

\begin{abstract}
Dual recycling is the combination of signal recycling and power recycling; both optical techniques improve the shot-noise-limited sensitivity of interferometric gravitational-wave detectors. In addition, signal recycling can reduce the loss of light power due to imperfect interference and allows, in principle, to beat the standard quantum limit. The interferometric gravitational-wave detector GEO 600 is the first detector to use signal recycling. We have recently equipped the detector with a signal-recycling mirror with a transmittance of $1 \%$. In this paper, we present details of the detector commissioning and the first locks of the dual-recycled interferometer.
\end{abstract}

\section{Introduction}

To date, all interferometric gravitational-wave detectors are basically Michelson interferometers optimised to measure tiny differential phase modulations of the light in the two arms. One of the main noise sources of these instruments is the photon shot noise. To reduce the effect of shot noise, the established technique of power recycling is used by all interferometric detectors. Power recycling allows to maximise the light power circulating in the interferometer arms without reducing the bandwidth of the detector or installing a larger laser. The sensitivity can be improved further by optimising the signal storage time, i.e. the average time for which the phase modulation sidebands induced by the gravitational wave are stored in the interferometer. For this purpose several advanced techniques have been proposed. For example, signal recycling in combination with power recycling was proposed by Meers 112 and is called dual recycling. It will be implemented in the GEO 600 detector from the beginning on and is planned for the second generation of interferometric detectors. GEO 600 is a British-German interferometric detector with $600 \mathrm{~m}$ long arms. Figure 1 shows a schematic diagram of the GEO 600 Michelson interferometer. The construction of the detector is complete and the Michelson interferometer is currently being commissioned 3 . 


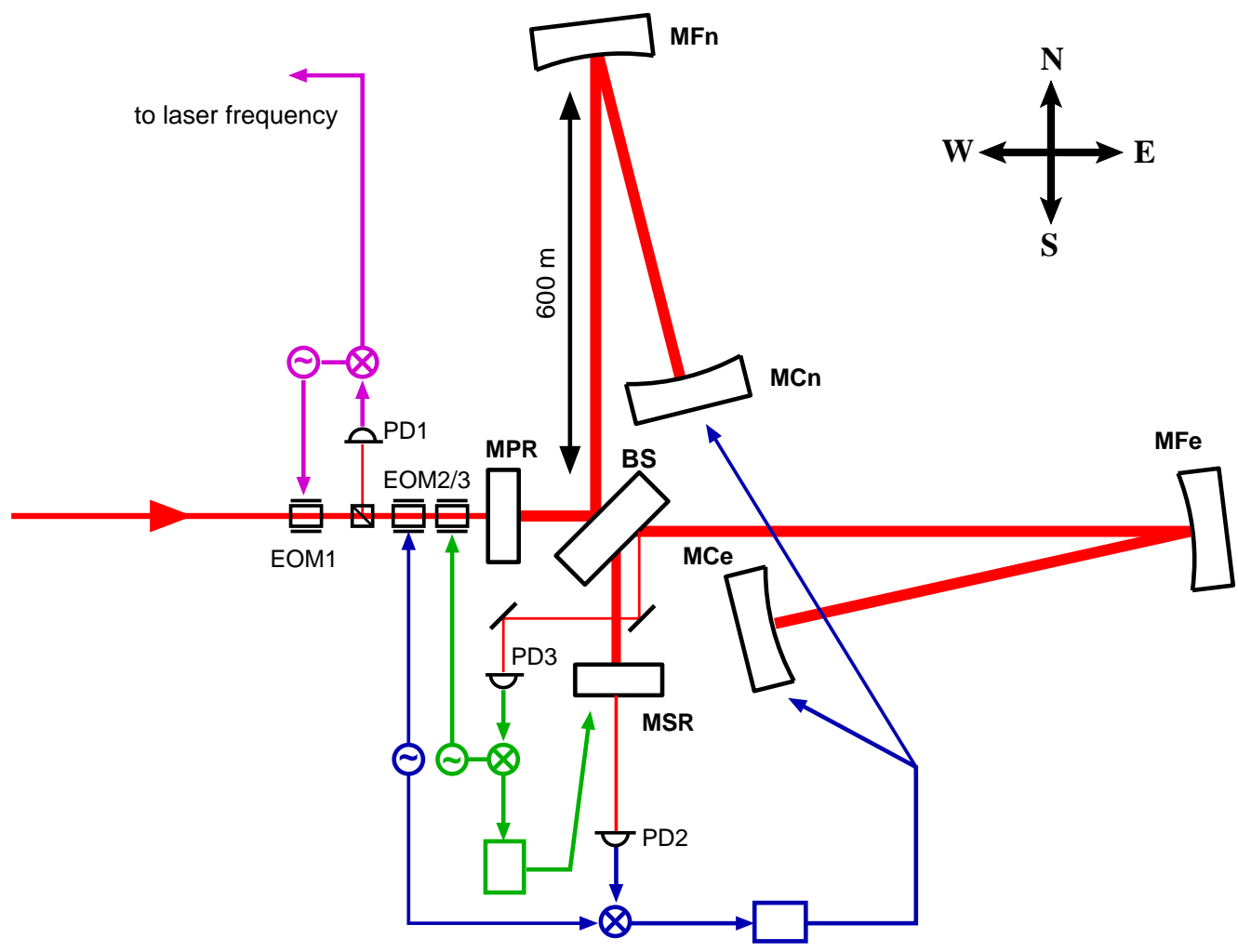

Figure 1: Simplified schematic of the GEO 600 Michelson interferometer. The beam enters the interferometer from the west through the power-recycling mirror (MPR) and is split at the main beam splitter (BS). Each arm of the interferometer is folded once by the folding mirrors (MFn and MFe) so that each arm has a round-trip length of $2400 \mathrm{~m}$. The folding is vertical but illustrated above as a horizontal setup. The end mirrors are MCn and MCe. Beams leave the Michelson interferometer at the west and the south ports. The output used for signal detection is the south port with the signal-recycling mirror (MSR). The three longitudinal degrees of freedom are controlled by three feedback loops, for which the laser light is phase-modulated before entering the interferometer. One pair of control sidebands (EOM1, frequency $f_{\mathrm{PR}} \approx 37.2 \mathrm{MHz}$ ) is used for controlling the power-recycling cavity via a standard Pound-Drever-Hall scheme (PR loop), which is part of the laser frequency stabilisation system. Two further pairs of control sidebands (at frequencies $f_{\mathrm{MI}} \approx 14.9 \mathrm{MHz}$ and $f_{\mathrm{SR}} \approx 9 \mathrm{MHz}$ ) are created with EOM2 and EOM3 to be resonant in the recycled interferometer. The error signal for controlling the operating point of the Michelson interferometer (MI loop) is obtained by demodulating the signal of the main photo diode PD2 in the south port (at $f_{\mathrm{MI}}$ ). Another photo diode PD3 is used to detect the light reflected by the AR coating of the beam splitter; the photo diode signal is demodulated at $f_{\mathrm{SR}}$ to generate the error signal for controlling the length of the signal-recycling cavity (SR loop).

The power-recycling technique exploits the fact that at the operating point dark fringe the Michelson interferometer (MI) behaves like a mirror for the injected light; the power-recycling mirror (MPR) recycles the light from the bright fringe in the west port, forming a cavity with the Michelson interferometer, the power-recycling cavity (PRC). This cavity is kept on resonance by a Pound-Drever-Hall method to resonantly enhance the carrier light inside the cavity. The maximum possible power enhancement is limited by losses in the interferometer. Assuming a power-recycling cavity with $T_{\mathrm{MPR}}, T_{\mathrm{L}} \ll 1$ we can write:

$$
P_{\text {cav }} / P_{\text {in }}=G_{\mathrm{PR}} \approx 4 T_{\mathrm{MPR}} /\left(T_{\mathrm{MPR}}+T_{\mathrm{L}}\right)^{2}
$$

where $T_{\mathrm{L}}$ is the power loss factor of one complete round-trip in the PRC and $T_{\mathrm{MPR}}$ the power transmittance of MPR. The ratio $G_{\mathrm{PR}}$ between the injected power and the intra-cavity power is called power-recycling gain. In the GEO 600 detector, the main origin of power loss is the imperfect interference at the beam splitter due to a mismatch of the beam phase fronts. Currently, this is caused mainly by a mismatch in the radii of curvature of the end mirrors. In the final state the effect will be dominated by the thermal lens in the beam splitter substrate. 
A passing gravitational wave (or an equivalent differential motion of the end mirrors $\mathrm{MCn}$ and MCe) will modulate the phase of the light in the interferometer arms and thus create 'signal sidebands' that are not directed to the west but to the south port. The power of the signal sidebands on the photo diode can be enhanced independently of the carrier light. The signal-recycling mirror (MSR) in the south port reflects the signal sidebands back into the Michelson interferometer. Again, the recycling mirror forms a cavity together with the Michelson interferometer, the signal-recycling cavity (SRC), which is tuned to resonantly enhance the signal sidebands. The maximum power enhancement of the signal sidebands on the photo detector, outside the SRC (over-coupled and $T_{\mathrm{MSR}} \ll 1$ ), is given by:

$$
G_{\mathrm{SR}}=4 / T_{\mathrm{MSR}}
$$

As the signal-recycling mirror closes the south port of the interferometer, it can considerably reduce the amount of carrier light power being lost through that port. FFT simulations have shown that the residual light power at the carrier frequency transmitted into the south port can be found almost entirely in second-order TEM modes (with respect to the eigenmode of the PRC). With MSR in place the SRC is (by design) not resonant for second-order TEM modes. Thus, these light fields are suppressed and the power loss from the PRC is reduced. This effect is also called mode healing. The FFT simulations have been used to predict the exact amount of the expected mode-healing effec 4 . In the case of the current GEO 600 detector and assuming negligible other losses inside the interferometer, we expect that the power-recycling gain can be approximated as:

$$
G_{\mathrm{PR}} \approx 4 T_{\mathrm{MPR}} /\left(T_{\mathrm{MPR}}+T_{\mathrm{MSR}} T_{1}\right)^{2}
$$

with $T_{1}$ as the power loss due to the phase front mismatch at the beam splitter. The mode healing effect also works for the SRC, so that MPR ensures a high finesse of the SRC.

\section{Control of the dual-recycled interferometer}

The dual-recycled Michelson interferometer has three longitudinal degrees of freedom: (1) the length of the power-recycling cavity, (2) the operating point of the MI (differential arm length), and (3) the length of the signal-recycling cavity (see Figure 11). The length of the power-recycling cavity is used as reference for the laser frequency stabilisation $\underline{5}$. Error signals for the two remaining degrees of freedom are obtained with the so-called Schnupp modulation technique (also known as 'frontal modulation' or 'pre-modulation'): The laser light is phase-modulated (at $\mathrm{RF}$ ) by an electro-optic modulator before it enters the interferometer. This creates two control sidebands that are injected into the interferometer together with the carrier. The beat note between the control sidebands and the carrier light leaking out of the Michelson interferometer generates a signal proportional to the Michelson interferometer's deviation from the dark fringe. The Schnupp modulation method can also be used to generate error signals with respect to other degrees of freedom. In particular, we use an additional Schnupp modulation at a different modulation frequency to generate a control signal for the SRC length.

With the power-recycling cavity and the Michelson interferometer being at their nominal operating points, the resonance condition inside the signal-recycling cavity is determined by the position of MSR along the optical axis. Each microscopic position of MSR corresponds to a different Fourier frequency of maximum enhancement. Therefore, a change in the position of MSR is also called tuning or detuning of the mirror and thus of the detector:

$$
\delta x_{\mathrm{MSR}}=\frac{\lambda}{2} \frac{f_{\mathrm{sig}}}{\mathrm{FSR}_{\mathrm{SRC}}}=4.23 \mathrm{pm} \cdot\left(\frac{f_{\mathrm{sig}}}{1 \mathrm{~Hz}}\right)
$$

with $\delta x_{\mathrm{MSR}}$ as the detuning of MSR, $\lambda$ the carrier wavelength, $f_{\text {sig }}$ the signal or detuning frequency and FSR $_{\mathrm{SRC}}$ the free spectral range of the SRC. The GEO 600 detector can easily be tuned to 


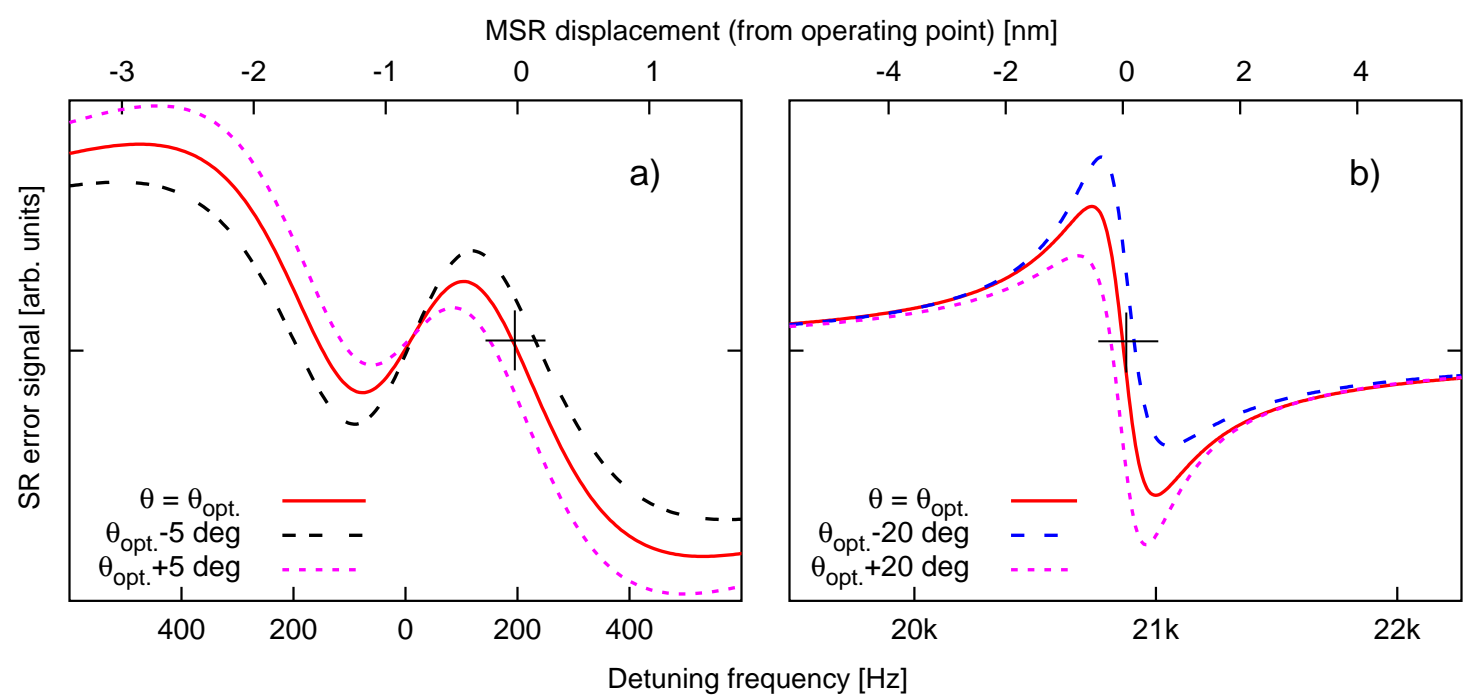

Figure 2: Simulated SR error signal for a) a typical detuning of $200 \mathrm{~Hz}$ that would be optimal for broadband operation and b) a large detuning of $\approx 20 \mathrm{kHz}$ (the operating points are indicated with a cross). Both graphs show the error signal for an optimised demodulation frequency and phase and for slightly different demodulation phases. It can be seen that the signal for $200 \mathrm{~Hz}$ detuning is suitable for controlling the MSR but not optimal for the lock acquisition process. The nearby zero crossing at $0 \mathrm{~Hz}$ requires that the control loop reduces any residual motion of MSR to well below one nm throughout all stages of acquisition. In addition, the signal is very sensitive to the demodulation phase. Using large detuning provides a symmetric signal and the residual motion of the MSR may be up to a few nm without causing the lock acquisition to fail. In addition, the dependency on the demodulation phase is also reduced. Both effects relax the requirements for the control loop and result in a more robust lock acquisition scheme.

a user-defined Fourier frequency by adjusting the modulation frequency and the demodulation phase of the SR loop and the gain of the electronic servos. We expect to continuously change the tuning of the detector during normal operation without losing lock of the system. A description of the general control concept is given elsewhere 6 .78.

\section{Commissioning of the detector}

During the commissioning of the GEO 600 detector we have first set up a power-recycled MI using a power-recycling mirror with $T_{\mathrm{MPR}} \approx 1.4 \%$ transmittance. The laser light power was reduced so that $1 \mathrm{~W}$ was injected into the main interferometer. The control systems of the power-recycled MI have been proven to work very reliably; during the S1 science run in summer 20029 , for example, the longest continuous lock of all subsystems lasted over 120 hours and the overall duty cycle was $>98 \%$. Recently, we have equipped the interferometer with a signalrecycling mirror having a power transmittance of $T_{\mathrm{MSR}}=1 \%$, which yields a detector bandwidth of $\approx 200 \mathrm{~Hz}$. First locks of the dual-recycled system, up to 15 minutes long, have been achieved. In addition, the interferometer can also be used in a power-recycled mode, simply by misaligning MSR.

Switching from power recycling to dual recycling requires a number of alterations to the control system. The modulation frequencies for the MI loop and the SR loop now have to be resonant in the 4-mirror resonator formed by MPR, MI and MSR. These frequencies are chosen to approximately match a multiple of the free spectral range of the power-recycling cavity:

$$
f_{\mathrm{MI}} \approx 14.9 \mathrm{MHz} \approx 119 \cdot \mathrm{FSR}_{\mathrm{PRC}}, \quad \mathrm{f}_{\mathrm{SR}} \approx 9 \mathrm{MHz} \approx 72 \cdot \mathrm{FSR}_{\mathrm{PRC}}
$$

An alternating sequence of measurements and simulations was used to determine the state of the detector and set the exact modulation frequencies and demodulation phases.

The expected SR error signals at nominal operating points have turned out not to be well suited for lock acquisition, see Figure 2. More symmetric and robust error signals can be 


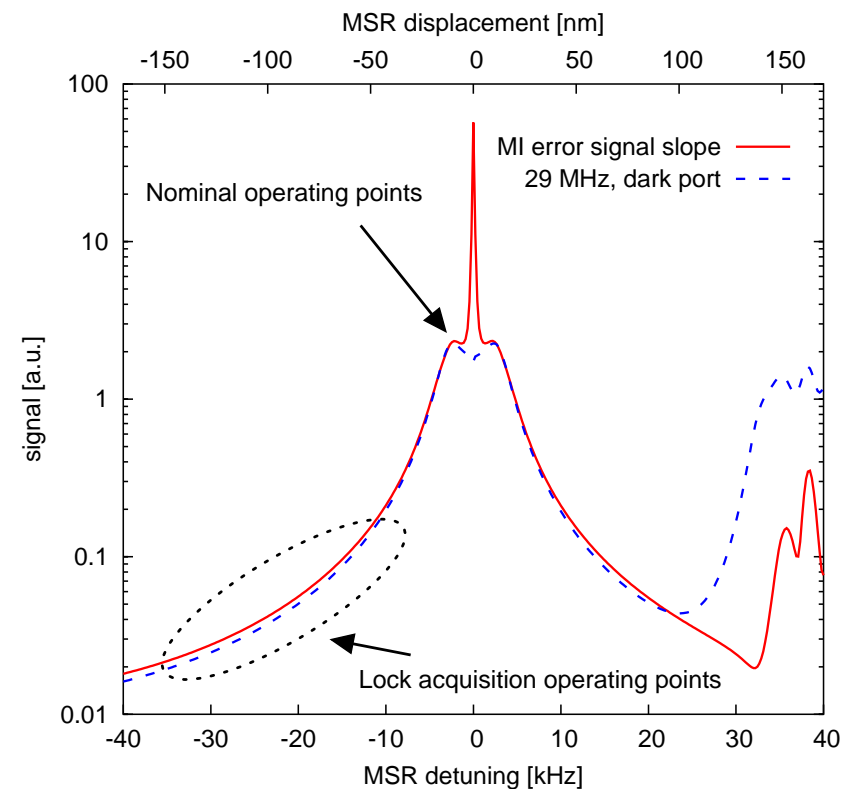

Figure 3: Simulated error signal slope of the MI control signal and a possible normalisation signal. The nominal operating points are at detunings between $0 \mathrm{~Hz}$ and a few $\mathrm{kHz}$. During lock acquisition we expect to use largely detuned operating points in a region between $-10 \mathrm{kHz}$ and $-35 \mathrm{kHz}$ (the complex structure for detunings $>20 \mathrm{kHz}$, a resonance of the second-order TEM modes in the SRC, will probably prevent lock acquisition for large positive detunings). The gain of the MI loop changes by more than three orders of magnitude when the MSR tuning is changed. A comparison of the available output signals showed that the power of the sidebands at $\approx 29 \mathrm{MHz}$ (the first harmonic of the MI control sidebands) detected in the south port can be used to determine the optical gain, except for very small detunings.

generated when the detector is largely detuned to frequencies between, e. g., $10 \mathrm{kHz}$ and $35 \mathrm{kHz}$. Therefore we decided to use such largely detuned operating points for the initial lock acquisition and then tune the detector while remaining in lock to its nominal operating point. For this purpose we plan to use an automatic gain control which takes the power of the $29 \mathrm{MHz}$ sidebands as a measure of the optical gain of the MI loop, see Figure 3 .

The three control loops (PRC, MI, SR) are subject to a natural hierarchy: First, a proper error signal for the MI exists only after the PRC has been locked to the laser light, whereas the PRC can be locked for almost all states of the MI by virtue of the asymmetric reflectance of the beam splitter. Second, a SR error signal can be obtained only after the MI is locked to the dark fringe whereas the position of MSR affects mainly the gain the MI error signal. These gain variations can be compensated using an appropriate normalisation signal (see below). Therefore, the lock acquisition scheme for GEO 600 has been designed as follows:

When the mode cleaners are locked and the laser light is present at the power-recycling mirror, the PR control closes the servo loop when the cavity is close to resonance. The optical gain of the PR loop depends on the state of the MI. In order to compensate for the gain changes, an automatic gain control is used: The PR error signal is divided by a signal proportional to the light power inside the cavity. When a PR lock is achieved, the MI control waits for the MI to pass through a dark fringe. A micro-controller is used to analyse several interferometer channels and to close the MI servo at a dark fringe if the differential mirror motion at that dark fringe is slow enough to be stopped by the force of the actuators. As soon as the MI is locked, the gain of this loop is increased to harden the control system against the influence of the still moving MSR. Finally, the SR control is switched on and closes the servo close to a resonance of the SRC.

The speed of the SR lock acquisition can be increased by using the following trick: The range of possible large detunings is relatively wide. Instead of setting the servo to one particular operating point (by adjusting the modulation frequency and phase), we use a micro controller to quickly sweep the operating point (by sweeping modulation frequency and phase). If the MSR position is somewhere in the required range, the controller will find it, determined by a well-defined zero crossing of the error signal, and thus set the nominal operating exactly to that position and close the servo loop.

Using the method described above we have achieved first locks of the dual-recycled detector. Figure 4 shows a measured time trace of several interferometer signals during a full lock acquisition. The acquisition time in this example was about 1 second. Typical durations of locks of 


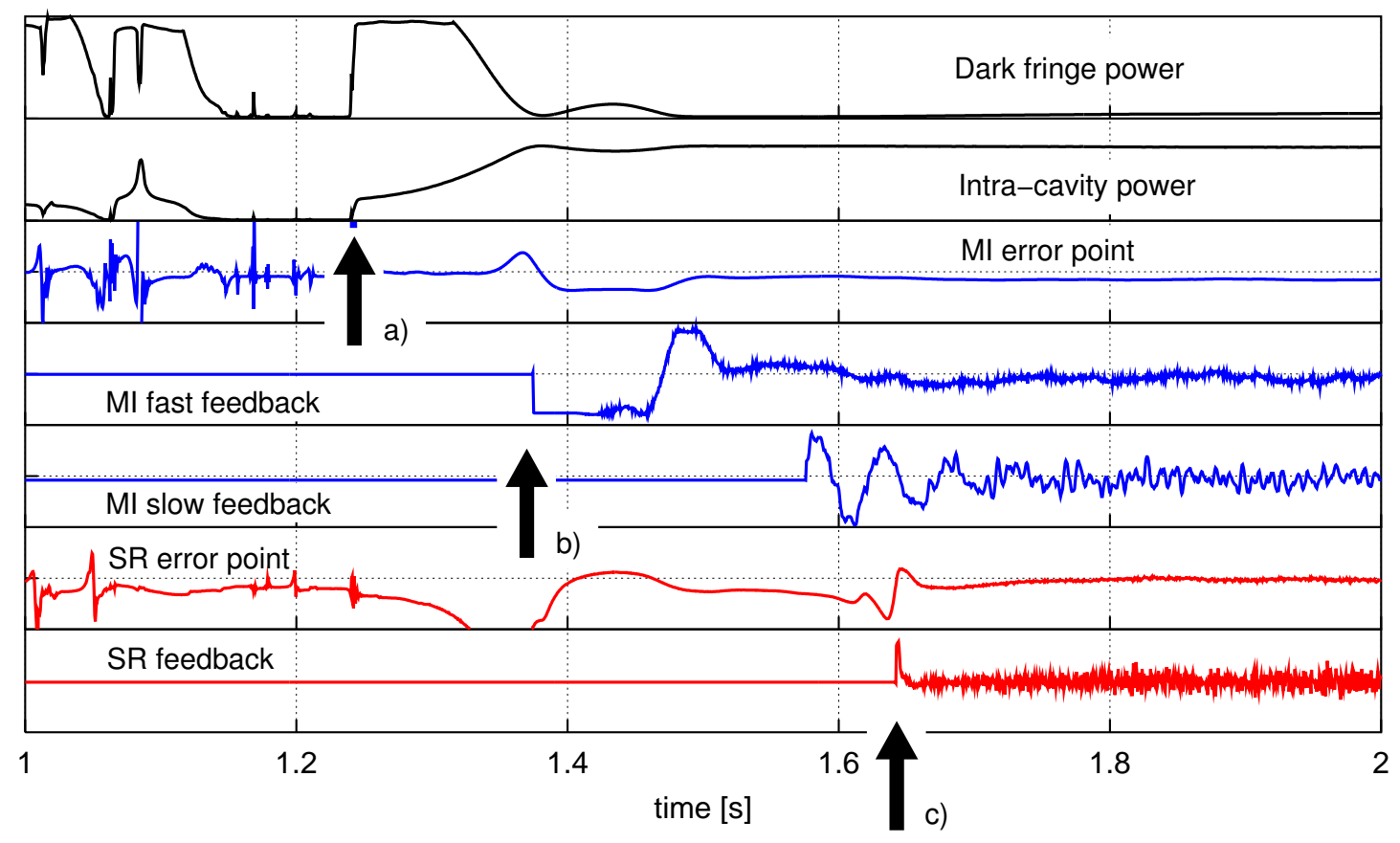

Figure 4: Measured time trace of interferometer channels during a full lock acquisition of the dual-recycled detector. The arrows indicate the time when the respective locks are achieved: a) the PRC is locked, light is injected into the MI which is, at that moment, not at the dark fringe; thus, the intra-cavity power is comparably low and the south port is bright. A tenth of a second later the MI passes through a dark fringe: the light power in the south port is reduced whilst the intra-cavity power builds up. Near the dark fringe the MI error point signal becomes visible. At its zero crossing the MI control loop is engaged, indicated by arrow b). First, the fast feedback (using an electro-static actuation on the test mass) is switched on. Some time later a slow feedback (via magnet-coil actuators at the intermediate mass) is switched on to increase the loop gain. In the following, the MI remains at the dark fringe while residual motion of the end mirrors is damped out. Only a few tenths of a second later the SRC passes through the SR operating point. The SR control loop is closed at the zero crossing of the SR error signal, marked by arrow c), and the SRC is locked.

the dual-recycled system currently range up to 15 minutes. We have also reached $\approx 300 \mathrm{~W}$ of power at the beam splitter (compared to $250 \mathrm{~W}$ without SR), which corresponds to the expected power increase due to the mode-healing effect.

\section{Acknowledgements}

The author would like to thank PPARC in the UK, the BMBF and the state of Lower Saxony in Germany.

\section{References}

1. B.J. Meers, Phys. Rev. D 38 (1988) 2317-2326.

2. K.A. Strain and B.J. Meers, Phys. Rev. Lett. 66 (1991) 1391-1394.

3. H. Lück et al., this issue.

4. R. Schilling, internal note (2002).

5. A. Freise et al., Class. Quantum Grav. 19 (2002), 1389-1397.

6. G. Heinzel et al., Phys. Rev. Lett. 81 (1998) 5493-5496.

7. A. Freise et al., Phys. Lett. A 277 (2000) 135-142.

8. G. Heinzel et al., Class. Quantum Grav. 19 (2002), 1547-1553.

9. M. Hewitson et al., submitted to Class. Quantum Grav.

10. A. Freise, http://www.rzg.mpg.de/ adf . 